\title{
Lévolution du contrôle législatif au Libéria
}

\author{
T. Debey Sayndee \\ Professeur agrégé et directeur, Kofi Annan Institute for Conflict \\ Transformation, University of Liberia
}

\begin{abstract}
Introduction
Vers la fin de 1989, la situation au Libéria a dégénéré pour s'enliser dans une guerre civile. Aucune unité des forces de sécurité nétait au service de la population. Bien que les combats armés, qui avaient duré 14 ans, aient finalement cessé en 2003, la réconciliation et la justice restent des objectifs lointains. Ce conflit civil violent ayant fait des troupes militaires une faction armée, mettre en place une force de sécurité républicaine axée vers le bien public est la première étape à franchir pour pouvoir reconstruire et faire prospérer le pays après le conflit. Le Libéria n’a plus confiance en ses forces de l'ordre et a besoin d'un secteur de la sécurité professionnel réglementé par un gouvernement démocratiquement élu et relativement indépendant du président. Autrement dit, il est temps de prévoir une transformation des forces de sécurité de l'Etat: au lieu de servir le régime en place, elles se préoccuperont désormais de la sécurité des citoyens.

Avec un passé de conflits et de régimes autoritaires tel que celui du Libéria, tout programme de réforme sérieux devrait intégrer la gouvernance démocratique de la sécurité comme principe fondamental. Le pays nest pas encore devenu une société ouverte dans laquelle tout un chacun a la possibilité de sexprimer, mais le processus de transition qui a commencé avec la fin de la guerre en 2003 est à présent bel et bien engagé.

Le présent chapitre prend la mesure des progrès réalisés par le Libéria en vue de transformer la dynamique de la gouvernance dans le secteur de la sécurité

Comment citer ce chapitre du livre:

Debey Sayndee, T. 2015. Lévolution du contrôle législatif au Libéria. Dans: Bryden, A et Chappuis, F (dir. publ.) Gouvernance du secteur de la Sécurité : Leçons des expériences ouest-africaines, Pp. 65-83. London: Ubiquity Press. DOI: http://dx.doi. org/10.5334/bav.d. Licence: CC-BY 4.0.
\end{abstract}


entre 2003 et 2013 à travers le prisme des événements qui ont déterminé l'évolution du cadre législatif de ce secteur. Avec la réforme dans son ensemble en toile de fond, il avance l'hypothèse qu'en se concentrant uniquement sur la formation et les équipements, les forces de sécurité libériennes nont pas soldé l'héritage d’années de conflit ni l'historique des contextes sécuritaires influencés par les différents régimes. En revanche, on imagine facilement que renforcer le contrôle du secteur de la sécurité dans le pays et instaurer une obligation de rendre compte aiderait à venir à bout de ces maux ancrés si profondément. Plus précisément, le chapitre examine l'importance du contrôle parlementaire pour encourager la gouvernance démocratique de la sécurité et sert de référence pour évaluer ce qu'il reste à faire par le Liberia dans ce domaine.

La première partie commence par une étude exploratoire des circonstances qui sous-tendent la dynamique de la gouvernance du secteur de la sécurité. Il présente l'historique des réformes, ce qui permet de mieux comprendre les changements déjà opérés dans l'organisation de la gouvernance de la sécurité au Libéria et de se familiariser avec les différents intervenants. La partie suivante traite des insuffisances liées à la gouvernance dans le processus de réforme et explique comment l'on pourrait commencer à mettre en place un contrôle public et législatif du secteur de la sécurité en partant d'un nouveau cadre législatif. La troisième partie analyse dans quelle mesure ces premiers pas sont un progrès vers une gouvernance démocratique plus durable. Pour finir, l'auteur explique les principaux enseignements tirés et quelles mesures adopter pour mettre en place une réforme du secteur de la sécurité.

\section{Les méthodes utilisées}

Cette partie porte sur deux approches à prendre en considération pour comprendre la réforme du secteur de la sécurité (RSS) au Libéria. La première est le contexte historique de la gouvernance politique dans le pays. La seconde est l'idée que la communauté internationale se fait de la RSS et la hiérarchie des priorités parmi les différentes activités concernées.

\section{L’historique de la gouvernance de la sécurité au Libéria}

Le Libéria a entamé une transition d'un régime autoritaire à une démocratie. Tous les efforts déployés par la communauté internationale sous légide des Etats-Unis ont abouti à la signature d'un Accord de paix global (Comprehensive peace agreement, Accra, 18 August 2003 - APG entre les différentes factions belligérantes en août 2003. L'événement a représenté un tournant décisif dans l'histoire du Libéria car il a mis un terme à un style de gouvernance autoritaire qui sétait enraciné depuis la création du pays en 1847. ${ }^{1}$ L'APG a été une victoire difficile à remporter car elle avait été précédée d’au moins 12 tentatives manquées pour désarmer les 
factions avant qu'une feuille de route n'ait été adoptée en vue d'instaurer la paix. En fin de compte, l'APG a marqué la fin du mandat de Charles Taylor en tant que président du Libéria et le début d'une ère nouvelle vers l'avènement d'une véritable démocratie. Après deux années d'un gouvernement provisoire entre 2003 et 2005, le pays a tenu ses toutes premières élections libres et honnêtes, qui ont amené au pouvoir la première femme présidente d'Afrique élue à l'occasion de la $52^{\mathrm{e}}$ législature du pays. Cette nouvelle tradition délections libres et honnêtes a perduré comme en témoigne la tenue d'une seconde série d'élections présidentielle et législatives après la guerre vers la fin 2011 lorsque la $53^{\mathrm{e}}$ législature est entrée en vigueur.

Cette transition de l'autoritarisme à la démocratie a également impacté le secteur de la sécurité. A cet égard il est important de comprendre comment le processus a démarré. Autrefois, le secteur de la sécurité était placé sous la responsabilité du cabinet du président de la République. Le président avait toujours exercé ses fonctions de manière autoritaire, voire même arbitraire, depuis l'époque des premiers colons et du parti libéral True Whig. Par exemple, le 18e président du Libéria William V. S. Tubman (1944-1971) a établi les règles du secteur de la sécurité, déclarant que «l'armée avait trois fonctions: obéir, obéir et obéir encore» (Bright 2002; cité dans Kieh 2008: 51). ${ }^{2}$ Son successeur était William R. Tolbert, Jr. (1913-1980), qui avait été le vice-président de Tubman pendant 19 ans et est devenu le $20^{\mathrm{e}}$ président du Libéria de 1971 jusqu’en 1980, année où il a été assassiné lors d'un coup d'Etat. Un événement important, les émeutes de la faim (Rice Riots) au début de 1979, provoquées par une tentative d'augmentation du prix du riz, produit alimentaire de base au Libéria, a contribué à renverser son gouvernement. Deux mille manifestants se sont regroupés pour une marche pacifique vers le palais présidentiel, mais la situation a dégénéré en émeute lorsqu'ils ont été rejoints par tout un groupe de «backstreet boys» - des jeunes sans abri, toxicomanes pour la plupart, vivant dans les rues de Monrovia. Le pillage généralisé qui a suivi a été jugulé grâce à un contingent de soldats venus de la Guinée voisine à la demande du président Tolbert. Ceci a provoqué un tollé au sein du secteur de la sécurité libérien, et fut l'élément déclencheur du coup d'état qui suivit.

Sous les régimes de Tubman, de Tolbert et de leurs prédécesseurs en remontant jusqu'en 1847, la politique de sécurité était élaborée puis appliquée par une instance centralisée œuvrant depuis le cabinet du président. On ne saurait trop insister sur le fait que la conception de la sécurité nationale en devenir au Libéria, guidée par des mesures législatives démocratiques et un contrôle du même type, se démarque totalement des politiques et des processus précédents. Pourtant, il reste des problèmes à régler en matière de gouvernance du secteur de la sécurité, notamment ceux liés à l'héritage d'un style patriarcal. Ce type de gouvernance dictatorial était en partie à l'origine des problèmes du secteur de la sécurité qui ont fait leur apparition plus tard, tels que la corruption et les menaces physiques envers les citoyens. 
L'existence de réseaux de corruption profondément ancrés et les clivages ethniques ont été les causes principales de l’effondrement du secteur de la sécurité durant la guerre civile et de la montée de la violence qui sensuivit. Les Libériens ont cherché à se distancier de ce passé pour évoluer vers une forme de gouvernance transparente et responsable qui placerait les droits des citoyens avant les intérêts personnels des détenteurs du pouvoir. Néanmoins, on trouve encore des institutions politiques et économiques défaillantes vestiges de l'ancien modèle à tous les niveaux de la société, y compris au gouvernement.

On constate tout de même quelques progrès, quoique lents et irréguliers, dans la mise en œuvre du processus de réforme. Par exemple, l'International Contact Group et le gouvernement ont imposé le Programme d'aide à la gouvernance et à la gestion économique en septembre 2005. Le Libéria a été le premier Etat à se conformer aux règles de l'Initiative pour la transparence dans les industries extractives et le premier pays d'Afrique de l'Ouest à voter une loi sur la liberté de l'information pour encourager une gouvernance plus transparente (Glencourse 2013). Récemment, le gouvernement a rejoint le Partenariat pour un gouvernement ouvert et s'est engagé à poursuivre une série d'objectifs ambitieux visant à obtenir une redevabilité accrue. Un grand nombre de ces mesures ont abouti grâce au président Sirleaf, mais le président précédent, le professeur Amos Sawyer qui a soutenu la cause de la gouvernance, a émis un doute sur la validité du raisonnement selon lequel «élire une «bonne» personne à la présidence suffirait à régler les problèmes (institutionnels)» (Sawyer 2002). Selon lui, le risque élevé et permanent d’abus de pouvoir est dû au fait que les problèmes des institutions politiques défaillantes nont jamais été abordés, notamment celui de la centralisation du pouvoir par la présidence.

\section{Une réforme de la sécurité sous l'égide de la communauté internationale}

La tentative guidée par la communauté internationale de refonder le secteur de la sécurité après la fin de la guerre a fourni loccasion de promouvoir la gouvernance démocratique de la sécurité et de prévenir toute nouvelle déstabilisation. Les organisations onusiennes ont très vite compris que la réforme du secteur de la sécurité était une priorité. Selon un rapport du Conseil de sécurité de l'ONU «l'une des raisons pour laquelle le pays est retombé dans la violence après la fin de la première guerre civile et les élections présidentielles de 1997 est qu'il n’y a pas eu de réforme des forces armées et de sécurité» (Conseil de sécurité des Nations Unies 2005a: par. 33; 2006: par. 6). Malgré des efforts considérables pour réformer la police et larmée, il n’a jamais été envisagé de s’engager sur la voie de la RSS. Cela explique en partie pourquoi les programmes de RSS ont été extrêmement ambitieux mais nont obtenu que des résultats mitigés (Malan 2008). Dix ans après avoir signé l'APG et malgré le soutien de nombreuses parties prenantes 
dans la région et au-delà, les perspectives d'un gouvernement légitime fondé sur les droits sont fortement menacées et la réforme, trop axée sur la police et les forces armées, sécarte de son objectif, à savoir la gouvernance démocratique du secteur dans son ensemble.

La RSS a commencé en 2004 avec la première initiative de réforme de la Police nationale libérienne (Liberia National Police - PNL) visant à former 1800 nouvelles recrues en prévision des élections de 2005, lancée par la Mission de maintien de la paix de l'ONU au Libéria (Mission des Nations Unies au Libéria - MINUL) à lépoque du gouvernement national de transition (Conseil de sécurité des Nations Unies 2005b: par. 30). Dix ans d'efforts pour réformer la PNL ont rendu les forces de police plus opérationnelles. Cependant, les comportements abusifs, la banalisation de l'impunité et la corruption endémique, dénoncés par Human Rights Watch dans son rapport d'août 2013, continuent déroder la confiance du public (HRW 2013). Actuellement, les citoyens ont de sérieux doutes quant à la compétence et à l'intégrité de la police (IRIN 2013). Tant que ces doutes subsisteront, notamment la suspicion de la participation de la police dans des actes criminels, la PNL ne pourra pas bénéficier des appuis dont elle a besoin pour être efficace, et l'autorité de l'Etat risque dêtre minée par les tentatives des citoyens de se protéger eux-mêmes de la criminalité et de la violence, par exemple en organisant des milices.

Conformément aux termes de l'APG, la réforme des forces armées du Libéria (Armed Forces of Liberia - FAL) a été fortement soutenue par les Etats-Unis. Pour mettre en route cette réforme, un contrat de sous-traitance a été passé avec la société de sécurité américaine DynCorp International, remplacé en 2010 par un accord de coopération avec l’armée américaine pour former et équiper les nouvelles forces armées. La Communauté économique des Etats d'Afrique de l'Ouest, le Ghana et le Nigéria ont également soutenu la formation de l'état-major des FAL. De ce fait, presque 2000 hommes triés sur le volet et bien entraînés sont venus grossir les rangs de l'armée libérienne (ICG 2009: 23). Parmi eux, seulement $5 \%$ étaient issus des anciennes FAL et en 2008 aucun groupe ethnique ne dépassait $15 \%$ (Malan 2008: 83). Le processus de vérification s'est particulièrement bien passé; deux experts qui y ont participé ont déclaré ne pas avoir vu de meilleure pratique dans le monde (ICG 2009: 12). Mais, même si les FAL avaient été établies sur de bonnes bases, le Secrétaire général des Nations Unies a signalé dans son rapport en 2009 «de sérieuses carences en matière de capacité» (Conseil de sécurité des Nations Unies 2009). Néanmoins, en 2013, la nouvelle armée libérienne a été jugée apte à participer à sa première opération de maintien de la paix au Mali et y a détaché une compagnie de 54 hommes. Depuis lors, cette opération a été renouvelée et même élargie. La mise sur pied au sein de l'armée d'un noyau central capable de gérer et de diriger les troupes est un processus interne qui doit être encouragé à la fois par le gouvernement et ses partenaires internationaux. Ainsi, malgré quelques faux-pas initiaux, il semble que les réformes de l’armée donnent de bons résultats. 


\section{Traiter les lacunes de la gouvernance}

Malgré ces maigres succès et même si les forces de la police et de l'armée se sont étoffées, aucun plan global pour instaurer la RSS n’a été mis en œuvre au Libéria. L'APG et les interventions extérieures qui ont suivi représentent une transition de la guerre vers une paix négative sans violence: Celle-ci est négative parce que, tandis que quelque chose d'indésirable a cessé d'exister, le résultat souhaité d'une plus grande justice sociale et d'un meilleur bien être social n’a pas été atteint (Galtung 1964: 1-4). Pour aller de l'avant, les Libériens ont dû se mettre d'accord sur les défis à relever et établir leurs propres priorités s'agissant de la réforme. Suite à cette prise de conscience, une série de débats publics a été organisée sur le thème de la sécurité nationale et un soutien a été apporté aux instances législatives durant la période d'après-guerre. Cette partie du chapitre rend compte des délibérations publiques sur la sécurité nationale et des efforts déployés pour renforcer la capacité législative en vue d'instaurer un contrôle du secteur de la sécurité en 2005-06, efforts qui préparé le terrain pour les modifications législatives des années suivantes dans le secteur de la sécurité.

\section{Les débats publics au sujet de la sécurité nationale}

En août 2005, et à nouveau en 2006, le ministère de la Justice et la MINUL ont animé conjointement des débats nationaux sur le secteur de la sécurité (DCAF 2005). Etant donné le passé autocratique de la gouvernance du secteur de la sécurité au Libéria, il n'y avait jamais eu de telles discussions en public avec une participation aussi diverse. L'idée à l'origine de cette nouvelle tendance était que débattre des besoins et des exigences de la RSS permettrait délargir le cercle des parties prenantes motivées pour créer une vision collective de la sécurité. Les participants étaient des Libériens issus d'horizons très divers et qui n’avaient pas joué de rôle dans lélaboration de la politique de sécurité nationale (notamment la législature provisoire, la sphère judiciaire et la société civile), de même que les ministères concernés, les organisations chargées de mettre en œuvre la réforme et des intervenants internationaux comme les Nations Unies. Ces débats ouverts et sincères ont permis aux Libériens de mieux définir les défis à surmonter pour instaurer la RSS dans le pays: ${ }^{3}$

- En ce qui concerne l'architecture du système de sécurité, la saturation de celui-ci vu le grand nombre d'instances de sécurité gouvernementales, de fonctionnaires, mais aussi de missions se chevauchant. A cela s'ajoutait le niveau douteux des compétences du personnel et la pratique du recrutement par favoritisme plutôt qu'en fonction de l'expérience;

- La nécessité de doter chaque institution du secteur de sécurité d'une mission claire et de faire en sorte que tous les groupes ethniques et religieux y soient représentés. Parallèlement, il faudrait veiller à ce que les personnels 
de ces organisations soient qualifiés, bien formés et recrutés selon une procédure transparente;

- Les violations flagrantes des droits de l'homme commises par des agents de sécurité et souvent restées impunies, par exemple des actes de torture, des arrestations arbitraires et des abus de pouvoirs à des fins d'enrichissement personnel. En l'absence d'une surveillance efficace, ces pratiques se sont généralisées. Il y avait plusieurs raisons à cela, notamment les pouvoirs excessifs du président ou le manque d'un véritable contrôle parlementaire. Les rémunérations insuffisantes ne faisaient qu'aggraver les choses car les personnels étaient obligés de recourir à la petite corruption et aux abus de pouvoir afin de subvenir à leurs besoins;

- L'importance de sécuriser les frontières du pays ainsi que le patrimoine de ressources naturelles, et la capacité à réagir impératifs de sécurité dans les provinces;

- Dernier point mais non des moindres, la nécessité d'adapter le coût du secteur de la sécurité aux moyens financiers dont le pays dispose, même si une aide internationale a été accordée au début.

Dans cet esprit, les Débats nationaux ont insisté sur le respect des lois et des droits humains. La participation de la société civile a été la bienvenue. La réforme de la constitution a également été évoquée. A cet égard, il était urgent de la réviser dans deux domaines: i) clarifier les rôles de toutes les instances gouvernementales afin d'éliminer les doublons en terme d'institutions de sécurité, et ii) éviter les éventuels abus d’autorité de la part du président qui, aux termes de la constitution, est investi du pouvoir de nommer pratiquement tous les dirigeants de l'appareil sécuritaire. En conclusion, bien que la communauté internationale soit prête à aider à mettre en place la RSS, la responsabilité principale de réformer le secteur de la sécurité et d'entamer le processus de reconstruction au lendemain des conflits incombe aux Libériens eux-mêmes. Cette prise de conscience s'est avérée prémonitoire car le degré d'appropriation locale de la RSS allait par la suite être contesté par la société civile et la législature, surtout s'agissant du soutien apporté par les Etats-Unis à la reforme de la défense.

\section{Le soutien à la législature}

Après les élections de 2005, il est rapidement devenu évident que la $52^{\mathrm{e}}$ législature était composée de $75 \%$ de novices (membres élus pour la première fois). Un groupe comprenant l'auteur de ces lignes a été créé pour renforcer les capacités de cette législature, notamment celles des Comités sur la sécurité et la défense de la chambre haute et de la chambre basse. Il a travaillé en collaboration avec le Réseau africain pour la réforme du secteur de la sécurité (African Security Sector Network), le Groupe conflit, sécurité et développement (Conflict Security and Development Group), le Centre pour la démocratie et le développement (Center 
for Democracy and Development), le Centre pour le contrôle démocratique des forces armées - Genève et d’autres encore. Il s'agissait de proposer une assistance holistique axée sur les besoins par des séances de travail, des travaux de recherche et du partage de connaissances (Jaye 2009: 7).

Ainsi, les membres des différents comités ont pu rencontrer des législateurs au Ghana et au Royaume-Uni et profiter de lexpérience de vétérans de la profession venus d'autres pays. Ces sessions ont permis aux législateurs libériens de mieux comprendre le processus du contrôle démocratique civil et comment rendre le secteur de la sécurité plus responsable. Les thèmes traités les plus importants étaient la mise en place d'un appareil sécuritaire à la fois unifié et différencié qui encourage l'entraide et évite le chevauchement des tâches sévissant dans les institutions existantes. Laccent était mis sur la séparation des rôles et des fonctions dans les diverses agences chargées de soutenir le secteur de la sécurité.

Exposer ces législateurs débutants à de nouveaux concepts et leur donner des conseils sur la manière de s'acquitter de leurs obligations a contribué à améliorer la fonction législative, surtout dans un contexte où l'eau, lélectricité et même le papier étaient rares. Il existe une corrélation évidente entre ces efforts pour améliorer la capacité de ces novices et les changements législatifs survenus par la suite dans le secteur de la sécurité. La loi de 2008 sur la défense nationale, rédigée par ces néophytes, s'est révélée être un pilier de la RSS. Cet instrument fondamental a également posé les jalons de la loi de 2011 sur la réforme de la sécurité nationale et sur le renseignement, laquelle est allée plus loin en établissant les principes de base pour les diverses institutions de sécurité au Libéria.

\section{La nouvelle loi sur la défense nationale}

En ce qui concerne la nouvelle loi de 2008 sur la défense nationale, le plus dur a été de définir les principaux rôles et responsabilités des FAL. Il s'agissait de démêler l'enchevêtrement des missions des nombreuses institutions de sécurité au Libéria. Déterminer le rôle de chacun des secteurs a été une tâche particulièrement ardue. Aucune des grandes institutions de sécurité ne disposait d'un énoncé de ses rôles et responsabilités, entre autres les FAL, la PNL, la police portuaire, la force d'intervention rapide, le service spécial de sécurité (appelé à présent service de protection des personnalités) et le bureau de l'immigration et de la naturalisation. Ce travail, entamé avec la rédaction d'une stratégie de sécurité nationale, n’a pu être finalisé qu’à partir de fin novembre 2007 (Conseil de sécurité des Nations Unies 2007: par. 134).

Le processus législatif a été achevé et la nouvelle loi sur la défense nationale a été adoptée en 2008. Elle remplaçait la précédente qui datait de 1956 et prévoyait une force professionnelle triée sur le volet et bien entrainée, mandatée spécifiquement pour assurer la protection de la souveraineté libérienne. A l'inverse, la loi de 1956 était inspirée d’une disposition obsolète du droit militaire 
américain imposant à tous les hommes un service militaire obligatoire et à tous les officiers une formation spécialisée. Le nombre de soldats nétait limité que par la capacité du gouvernement à « fournir un abri, des moyens de subsistance, un uniforme [sic], des armes et des munitions, et des soins hospitaliers». Ce modèle de service militaire a contribué à mettre sur pied une armée imposante qui a été par la suite démobilisée dans le cadre du processus de réforme. Cependant, aucun dispositif d’assistance adapté n’a été prévu pour les anciens combattants, comme on va le voir ci-dessous.

A loccasion de la révision de son concept du service militaire et de la gestion des effectifs, la nouvelle loi de 2008 sur la défense nationale a ajouté des dispositions selon lesquelles le pays devrait travailler avec les Etats-Unis afin de développer son secteur de la sécurité et de bénéficier de l’aide apportée par d'autres partenaires internationaux aussi bien d'Afrique que d'ailleurs. Jusquà présent ceci a permis au Libéria d’obtenir l’appui de pays comme le Ghana, le Nigéria et la Chine, qui se sont chargés de former des cadres moyens et supérieurs. Cette réorientation a une incidence sur tous les secteurs de l'appareil sécuritaire. Des personnels de niveaux supérieur et intermédiaire ont été choisis pour suivre une formation dans ces pays et ont commencé à intégrer les meilleures pratiques dans les méthodes opérationnelles. Grâce à ces efforts collectifs, le Libéria a au moins pu tirer quelques avantages d'une coopération SudSud et d'un partage des expériences. Cette initiative pour élargir la formation des personnels de sécurité libériens en les envoyant à létranger représente un tournant radical par rapport à l'utilisation exclusive de méthodes de formation américaines particulièrement après 2003 et en général depuis la création de la république.

D’après la loi de 2008 sur la défense nationale, la mission première des FAL est de protéger la souveraineté du pays ainsi que son intégrité territoriale. Outre ce mandat, les FAL doivent aussi mener des opérations de maintien de la paix et apporter un soutien humanitaire aux autorités civiles en cas de catastrophe. Larticle 2.3 e) stipule que les FAL sont tenues de venir en aide aux services de maintien de lordre lorsque la situation l'exige et que le président a donné son aval. Mais, elle stipule aussi qu'en temps de paix, les FAL ne doivent pas participer aux activités de police et qu'elles ne peuvent intervenir qu'en dernier ressort, quand la menace dépasse les capacités de réaction des services d’ordre. A l'article 2.5, il est stipulé que les FAL doivent exercer leurs obligations de façon impartiale. Toutes ces dispositions ont été formulées pour pallier les difficultés que les FAL ont connues durant le conflit civil lorsqu'elles se sont trouvées embourbées dans la guerre.

Toutefois, la loi de 2008 sur la défense nationale n’a pas réglé certains problèmes inhérents au passé récent des FAL. Ainsi, léchec de laction en justice intentée par plus de 200 soldats à la retraite et les veuves de soldats morts au combat est la preuve que les mauvaises relations entre les civils et les militaires sont toujours d’actualité. Les plaignants arguaient dans cette requête, rejetée 
par la cour en novembre 2013, que le gouvernement avait dissous les FAL en violation de la constitution et n'avait pas accordé de prestations de retraite (Parley 2013; Gbelewala 2013). Les anciens combattants soutenaient qu'au lieu de réorganiser les FAL, comme prévu dans l'APG, le gouvernement avait dissous l'armée sans aucun régime de retraite. De tels litiges montrent bien les faiblesses du processus de désarmement, démobilisation et réinsertion au Libéria.

Malgré ces insuffisances, la loi de 2008 sur la défense nationale a tout de même permis d'établir de nouvelles bases législatives pour la séparation du maintien de l'ordre et de la défense nationale dans le cadre d'un contrôle démocratique civil, de l'Etat de droit et du respect des droits humains. Une fois les rôles et responsabilités des forces de défense clairement inscrits dans la loi, élargir ce raisonnement à d'autres secteurs de la sécurité est devenu possible. C'est d'ailleurs ce que la loi sur la Réforme de la sécurité nationale et sur le renseignement (National Security Reform and Intelligence Act 2011 - loi RSNR), adoptée en 2011, a cherché à faire.

\section{La loi de 2011 sur la réforme de la sécurité nationale et sur le renseignement}

La loi RSNR tant attendue a été très controversée car elle a entraîné la fermeture de nombreuses institutions du secteur de la sécurité afin de répartir les services de sécurité nationale entre un nombre d'organismes plus petit et plus facilement gérable. La loi a été soumise à l'approbation du président le 30 août 2011 et a fait l'objet de nombreux allers-retours avant d'aboutir à deux amendements. Par exemple, le ministère de la Sécurité nationale a été supprimé dans la première version, mais rétabli ensuite par un amendement. Réduire le nombre des institutions de sécurité était en quelque sorte considéré comme un moyen de réduire les coûts et la complexité et de se débarrasser du modèle de gouvernance autocratique hérité du passé, dans lequel les intérêts des institutions de sécurité sopposaient à l'avantage du président. Pour d'autres en revanche, cela revenait à placer trop de pouvoir entre les mains de quelques rares institutions incontournables tout en surchargeant les institutions émergentes encore fragiles d'un surcroît de tâches, laissant la nation incapable de faire face à de nouvelles menaces. De nombreux groupes de pression se sont manifestés pour éliminer les institutions réputées avoir servi les intérêts d'élites politiques rivales. Les opinions restaient très partagées quant à la façon de percevoir ces institutions. Plusieurs séances réunissant à la fois des membres du pouvoir exécutif et du pouvoir législatif ont tenté d'aboutir à un compromis. La loi RSNR n'a été finalisée qu’en mai 2013, mais semble encore être «en cours d'élaboration» eu égard aux nombreux obstacles à surmonter et aux problèmes à résoudre encore en suspens (Libéria 2011).

La loi RSNR de 2011 s'est en effet avérée être "en cours d'élaboration». En 2013, plusieurs amendements importants ont été ajoutés, visant à revoir 
l'appareil de sécurité initialement établi. ${ }^{4}$ La loi de départ s'est enrichie de deux nouveaux chapitres rétablissant le ministère pour la Sécurité nationale (Ministry of National Security - MSN) et réactivant le bureau national des enquêtes (National Bureau of Investigations - BNE). Les institutions de sécurité reconnues par la dernière mouture de la loi RSNR comme faisant partie intégrale de l’appareil de sécurité nationale sont:

- la police nationale du Libéria;

- le bureau de l'administration pénitentiaire et de la réinsertion (ministère de la Justice);

- le ministère de la Sécurité nationale;

- le bureau de l'immigration et de la naturalisation;

- le service de la répression du trafic de stupéfiants;

- le bureau des douanes et des droits indirects;

- le bureau national des enquêtes;

- le service national de lutte contre les incendies;

- l’agence pour la sécurité nationale;

- le service de protection des personnalités (appelé auparavant service spécial de sécurité).

Le MSN et le BNE ont récupéré quelques unes de leurs anciennes fonctions et responsabilités précédemment assignées à la PNL et l'agence pour la sécurité nationale par la loi RSNR de 2011. Le MSN était chargé d’organiser et de valider des données fournies au gouvernement par différents services du secteur de la sécurité puis de les coordonner et de les partager avec les agences concernées, telles que l'agence pour la sécurité nationale. La lutte contre la cybercriminalité et d'autres activités criminelles high tech font aussi partie des attributions du MSN, qui informe également le président au sujet des activités des autres agences de sécurité. Quant au BNE, il enquête sur les crimes graves comme les meurtres, les incendies criminels, les entrées illégales au Libéria et le vol des biens de l'Etat. C'est le BNE qui conserve et tient à jour le fichier des criminels connus et communique les renseignements à la PNL et d'autres agences concernées.

Ces amendements à la loi RSNR initiale de 2011 témoignent des défis auxquels les services de sécurité et de renseignement doivent faire face aujourd'hui. Alors que les obligations de ceux qui font partie du cercle fermé des initiés aux «secrets d'Etat» sont clairement mentionnées, il n’existe aucun mécanisme précis (à part la supervision générale de la législature, du président et de l'exécutif) empêchant le MSN, le BNE et l'agence pour la sécurité nationale d’outrepasser leurs pouvoirs. Ceci est dû au fait que le rôle du système judiciaire n'est pas mentionné dans la loi RSNR initiale ni dans ses amendements. Toute personne qui se considère accusée à tort d'un délit n’a aucun moyen de faire appel et ne dispose d'aucun recours. 
Ainsi, bien que le contrôle civil soit un principe fondateur de la loi RSNR, il n'a pas été concrétisé dans des mécanismes spécifiques (PBC 2012: par. 35). De même, les personnes concernées par cette loi ne sont pas suffisamment outillées pour s'acquitter de leurs tâches et ont besoin d'une formation plus approfondie. Il faut faire un effort supplémentaire pour améliorer le contrôle civil, resserrer les liens avec la communauté, réduire la corruption et se doter des ressources nécessaires, sans quoi les plans tels qu'ils sont actuellement décrits dans la loi RSNR pourraient être contrariés (PBC 2012: par. 35).

\section{Les progrès accomplis et les défis à relever}

Ce qui précède montre que la législature libérienne est au cœur du processus de RSS et que, pour la première fois, elle participe activement à la mise en œuvre de la gouvernance du secteur de la sécurité durant la transition vers la démocratie. Comme l’a déclaré Thomas Jaye, pour garantir l'efficacité de la réforme du secteur de la sécurité au Libéria, il faut renforcer le contrôle législatif dans trois domaines: l'autorité, la capacité et l'état d’esprit (Jaye 2009, 9). De ce point de vue, il reste encore de nombreux problèmes à traiter tels que la suprématie de l'exécutif, le manque d'intégrité chez certains législateurs et la corruption.

Il existe bien un cadre fonctionnel régissant le contrôle législatif même si le président détient une grande partie des pouvoirs constitutionnels: l'article 43 de la Constitution libérienne confère au Parlement des pouvoirs étendus pour traiter toutes les questions de sécurité (Ebo 2005: 23). De plus, la législature opère de telle façon qu'elle permet la création de comités sur la sécurité et la défense, qui pourraient superviser le secteur de la sécurité par le biais d’audiences et par la convocation de témoins. Le processus de sélection des membres des comités ayant été élargi, ils ne sont plus nommés par le seul président de l'assemblée. La législature fait ainsi preuve de davantage d'ouverture et se prépare mieux à faire du bon travail.

Les efforts déployés en faveur de la transparence, comme l'Open Budget Initiative, ont renforcé davantage l'influence du corps législatif sur la gouvernance du secteur de la sécurité, en rendant publiques les crédits accordés à l’armée et aux autres institutions de sécurité. Soumettre le budget de la sécurité à l'examen du public est une mesure susceptible d'accroître sa confiance, laquelle ne pourra quaugmenter en présence d'un contrôle plus strict. Cependant, l'inconvénient de ce système est le déficit budgétaire permanent autorisant l'exécutif à réallouer des fonds sans l'aval du législatif. Ce dysfonctionnement est encore plus grave en situation d'urgence et de menace sécuritaire.

Toutefois, l'autorité n'est pas la seule variable déterminant le contrôle législatif. Encore faut-il que les législateurs puissent être en mesure d'utiliser les pouvoirs à leur disposition. La complexité et surtout le culte du secret, habituel dans les milieux de la sécurité, représentent de sérieux obstacles. Les responsables se retranchent souvent derrière le soi-disant «intérêt de sécurité national» afin 
de limiter l'influence des législateurs. Une autre complication est le renouvellement du personnel. Le temps que les législateurs se familiarisent avec les questions de défense, de sécurité et de renseignement et acquièrent de l'expérience, ils sont affectés à un autre comité ou alors leur mandat se termine. Pour finir, il $\mathrm{y}$ a le problème du niveau deéducation généralement bas parmi les législateurs.

La capacité, la confidentialité et le renouvellement du personnel sont des facteurs étroitement liés. La capacité concerne non seulement le niveau déducation mais aussi l'intégrité personnelle. La culture du secret est, au Libéria, une variable culturelle négociable, autorisant tous les compromis. Enfin, avec le temps, il est probable que le renouvellement diminuera au fur et à mesure que les électeurs feront confiance aux représentants choisis par eux et qu'ils les éliront à nouveau dans d’autres scrutins sur la base de cette confiance plutôt que de s'en remettre au «choix officiel». Un défi de taille lors des prochaines élections sera de renforcer le rôle et la participation de la population dans ce processus.

Le dernier facteur permettant de renforcer l'efficacité du contrôle du secteur de la sécurité est létat d’esprit. Dans le passé, la législature libérienne a toujours cédé aux exigences de l'exécutif, mais la situation a changé. Les comités concernés revendiquent leur utilité s'agissant de la sécurité et n’hésitent pas à demander aux ministres de s'expliquer. Ainsi, ce tout nouveau rôle du corps législatif devrait conférer une légitimité à l'appareil sécuritaire de l'Etat tout en renforçant la position de la législature vis-à-vis de lexécutif.

La question de létat d'esprit ne se retrouve pas seulement dans le contrôle de la législature. L'exécutif doit lui aussi respecter le rôle des législateurs pour garantir la gouvernance démocratique du secteur de la sécurité, ce qui n’a pas toujours été le cas au Libéria. Depuis l'indépendance, la volonté et l'engagement du pouvoir législatif à assumer ses fonctions de supervision ont été entravés par le régime du parti unique et par le style souverain de la présidence. A cause de ces deux facteurs, la législature s’est toujours pliée à la volonté de l'exécutif, et la loyauté politique a miné l'indépendance du Sénat et de la Chambre des représentants. Mais la situation est en train de changer. Même si le président est investi de pouvoirs considérables par la constitution, le corps législatif a commencé à se prévaloir de son autorité prévue par la loi pour discuter des décisions entérinées auparavant sans discussion par ses prédécesseurs.

\section{Pour un changement durable du secteur de la sécurité au Libéria}

Elargir davantage les débats publics, établir un nouveau fondement légal pour la sécurité nationale et renforcer la capacité législative représentent des changements sans précédent dans la gouvernance du secteur de la sécurité au Libéria. Pourtant, ces initiatives ne sont que les prémices d'un long cheminement vers une véritable gouvernance démocratique. Elles s'inscrivaient dans le contexte 
plus large des efforts déployés par la communauté internationale pour régler les aspects techniques de la réforme, mais elles nont pas assez tenu compte des questions spécifiques à la gouvernance. Il s'agit donc d'évaluer dans ces lignes dans quelle mesure la RSS a le pouvoir de changer le paradigme actuel du Libéria en un modèle durable, centré sur ce même processus de RSS.

\section{Léquilibre entre l'appropriation locale et l'intervention de la communauté internationale}

Le processus de RSS s'articule autour de plusieurs intervenants. Le Libéria étant un Etat failli, l'implication de la communauté internationale était - et est toujours - considérée comme nécessaire pour renforcer la capacité des institutions de sécurité. Le pays a donc été obligé d’entreprendre sa RSS en acceptant une aide extérieure conséquente, dont la portée considérable s'appliquait exclusivement à l'exécutif. Ainsi, eu égard à l'appropriation locale et la participation démocratique, toutes les parties prenantes à l'exception de l'exécutif ont été écartées du processus de RSS, notamment au début (Onoma 2014; Ebo 2008; Loden 2007).

L'exemple le plus flagrant est l'accord que les Etats-Unis ont passé avec une entreprise privée afin de mettre en œuvre les réformes de défense pour le compte du Département d'Etat américain. La société en question, DynCorp, a refusé de rendre des comptes au Parlement libérien concernant la refonte des FAL, invoquant des obligations contractuelles envers ce même département. Du fait que la réforme était sous-traitée, il était extrêmement difficile pour le corps législatif et la société civile d'exercer un contrôle quelconque et encore moins de participer au processus. Les caractéristiques, la composition et les spécificités des nouvelles forces armées du Libéria ont donc été déterminées sans l'avis du Parlement ni celui de la société civile, ce qui montre à quel point ils sont exclus du processus de RSS. Des représentants du gouvernement et des groupes de la société civile ont exprimé leur inquiétude à ce sujet et ont demandé que le processus soit «libérianisé ».

La société civile devrait jouer un rôle de premier plan dans le nouveau secteur de la sécurité comme cela a été souligné lors des débats nationaux et du processus délaboration législatif qui sest ensuivi. Renforcer ses fonctions de supervision encouragerait également l'adhésion de la population. Dans le passé, la société civile était tenue informée, mais non consultée. Il va falloir élargir son rôle au-delà des quelques actions précédemment citées. La société civile était explicitement mentionnée à l'article VII (3) dans la section de l'APG relative au renforcement du contrôle civil et à la participation locale dans la RSS au Libéria. Grâce aux processus de gouvernance élargis, elle a pu disposer d'une première tribune limitée. Plusieurs équipes œuvrant dans des domaines liés à la sécurité se sont mobilisées pour former un Groupe de travail sur la RSS, qui est devenu particulièrement actif à partir de 2008. Des forums communautaires 
ont été organisés afin d’encourager la participation de la société civile et du public dans les activités policières, bien que cette initiative ait connu plusieurs déboires avant dêtre finalement relancée en 2011 pour devenir le programme patrouilles de quartiers.

Bien que la participation de la société civile soit plus importante aujourd'hui quà aucun autre moment dans l'histoire du Libéria, ces initiatives n’ont qu'un impact limité. Ceci est dû au fait que la société civile n’a pas participé au processus de réforme depuis le début. Sa mise à lécart montre à quel point il est courant de penser qu'efficacité ne va pas forcément de pair avec légalité, transparence et responsabilité (Bendix et Stanley 2008: 27). Ce manque d'implication a sans doute aussi été à l'origine de la décision des Américains de couper leurs liens avec DynCorp pour se charger directement de la formation des FAL par le biais de lopération Onward Liberty, un programme d'une durée de cinq ans. Cet accord de coopération a permis de «libérianiser» le commandement par le biais de promotions rapides et finalement la nomination en 2014 d'un chef détat-major libérien en remplacement d'un général nigérian qui avait occupé ce poste par intérim.

\section{Nouvelles opportunités de contrôle démocratique}

Après la promulgation de la loi de 2008 sur la défense nationale, une autre initiative a été la Commission de réforme de la gouvernance (Governance Reform Commission - CRG), devenue permanente en 2009 sous le nom plus simple de Commission pour la gouvernance. La CRG a été initialement créée conformément aux dispositions de l'APG en tant quorgane provisoire mandaté pour dispenser ses conseils concernant les questions de bonne gouvernance. Elle a donc participé activement à l'élaboration des premières réformes et a joué un rôle de premier plan dans les débats nationaux depuis 2006 pour se voir finalement confier la tâche de rédiger des projets de réforme par le président. ${ }^{5}$ Celui-ci avait d'ailleurs déjà eu recours à ses conseils et son influence pour des questions d'intérêt national comme la sécurité avant d'officialiser ses fonctions en 2009 (Libéria 2006). La CRG a réalisé une évaluation du secteur de la sécurité et a revu la législation existante. Elle est arrivée à la conclusion que le processus de RSS «se déroulait dans un contexte (interne et externe) très instable, incertain et fragile» (Jaye 2006). Selon elle, la centralisation du pouvoir entre les mains du président, l'absence d'un système judiciaire indépendant et la règle du parti unique qui prédominait antérieurement étaient autant d’obstacles à surmonter. La loi RSNR de 2011 a représenté un pas important vers l'instauration du type de RSS souhaité par la CRG (Jaye 2009).

D’autres organismes indépendants mis en place pour renforcer le fonctionnement de la RSS sont: i) la Commission anticorruption créée en août 2008 aux termes d'une loi permettant de mener des enquêtes, poursuivre les responsables, prévenir la corruption, sensibiliser le public à ce fléau et aux bienfaits de 
son éradication (Conseil de sécurité des Nations Unies 2009: par. 2); et ii) la Commission nationale indépendante sur les droits de l'homme, telle qu'exigée par l'article XII de l'APG et créée par la Commission Vérité et réconciliation en vue de mettre en œuvre ses recommandations (ICTJ 2015). Ces deux initiatives sont positives et porteuses de promesses, mais leur importance à long terme dépend de la façon dont elles sont mises en œuvre car les résultats sont pour l'instant mitigés.

Ces dix dernières années, le monstre à plusieurs têtes truffé de redondances quétait l'appareil sécuritaire libérien s'est mué en un dispositif cohérent dans lequel les limites des attributions et des responsabilités sont clairement définies. Ceci est en partie dû aux efforts mentionnés précédemment et déployés pour renforcer la capacité législative, ainsi qu’au cadre légal, résultat de ces efforts. Lélaboration de ce cadre est pratiquement terminée avec la promulgation en 2011 de la loi RSNR et celle de ses amendements en 2013.

Pour mettre en place la RSS, il reste encore à introduire plusieurs textes de loi: une loi sur la police, une réforme de l'agence de lutte contre le trafic de stupéfiants, la loi sur la stratégie de défense nationale et le contrôle des armes à feu. En outre, d'autres sujets doivent être abordés comme la relation entre la sphère sécuritaire et la sphère politique, qui doit être plus claire de façon à éviter la politisation de la sécurité, ou les modalités de nomination des hauts fonctionnaires, qu'ils soient politiques ou non.

\section{La réforme de la police}

La confiance du public ayant été gravement entamée par suite de l'inefficacité chronique des forces de lordre, il semble urgent de promulguer une nouvelle loi sur la police. Certes, comme l'a déclaré Thomas Nah du Centre libérien pour la transparence et la redevabilité (Stroehlein 2013), «même si les armes se sont tues ces dix dernières années, cela ne veut pas dire que tout va bien ici». D’après lui, «le Libéria ne pourra pas avancer tant que la police ne changera pas» (Stroehlein 2013). Même si l'on admet que la corruption est généralisée dans toutes les institutions du pays, les abus constatés dans la PNL sont particulièrement flagrants car comme le dit Cecil Griffiths, président de l'Association pour le maintien de lordre au Libéria, elle est censée représenter l'Etat». ${ }^{6}$ Etant donné ses ressources et sa complexité, il est indispensable de la contrôler (Gompert, Davis et Stearns Lawson 2009; Gompert et al. 2007).

Récemment, un service de contrôle interne de la PNL a été créé, la Division des normes professionnelles (Professional Standards Division). Depuis qu'il est opérationnel, les officiers de la PNL sont plus enclins à dénoncer les comportements abusifs des autres officiers. Cependant, Human Rights Watch et d’autres organismes similaires ont insisté pour que le gouvernement et la PNL mettent en place un comité de supervision civil indépendant destiné à traiter les plaintes relatives à des comportements répréhensibles. 
Il est évident qu'il va falloir prévoir une nouvelle loi sur la police et qu'elle devra être strictement appliquée. Il existe plusieurs possibilités à cet égard. Bien que cela soit difficile à mettre en œuvre, elle pourrait faire partie d'un ensemble fourre-tout sur la sécurité. Une autre solution serait d'actualiser le décret relatif à la PNL de 1975 (Jaye 2008: 169). Les dispositions à prévoir devraient concerner les procédures d'arrestation et de détention. En l'absence d'un système efficace, équitable et transparent pour réglementer les arrestations, les procès et les incarcérations, les personnes inculpées pourraient se retrouver en prison sans avoir été jugées ou au contraire être remises en liberté sans qu'il y ait eu de poursuites (Gompert et al : 57). Ceci est, malheureusement, la réalité au Libéria aujourd'hui. Aux termes d'une telle loi, la PNL serait subordonnée à l'autorité et au contrôle du ministère de la Justice et les services de police auxiliaires seraient incorporés à la PNL (Gompert et al : 77).

Cette loi exhaustive sur la police viendrait compléter la loi de 2008 sur la défense nationale en ce qui concerne les FAL, et permettrait à la PNL de redorer son blason et de remplir sa mission. C'est une pierre angulaire de l'édifice libérien de la sécurité nationale, composante dont l'absence rappelle les responsabilités cruciales que le législatif devra endosser relativement à la RSS.

\section{Conclusion}

La lente descente dans la crise, puis les 14 années de guerre civile intermittente qui ont suivi, ont été dévastatrices pour le pays et sa population. Faire renaître du chaos un secteur de la sécurité viable a été une tâche titanesque. Pourtant, en dix ans depuis la signature de l'APL, le Libéria a réussi à reconstruire son armée et à mettre sur pied une nouvelle force de police fondée sur des bases techniques plus solides et mieux outillée pour protéger ses citoyens, les frontières du pays, et même participer à des opérations de maintien de la paix.

Cette initiative lancée par la communauté internationale pour améliorer l'efficacité du secteur de la sécurité a cependant échoué dans ses efforts pour rallier la sphère publique au processus de réforme. Le Libéria n’a pas su récolter les fruits des améliorations qu'il a apportées au secteur de la sécurité, car il ne s'est pas préoccupé de renforcer parallèlement la redevabilité démocratique. Comme le montre ce chapitre, les incohérences et «les pièces manquantes» du cadre légal libérien pour la sécurité nationale restent des obstacles. La suprématie de l'exécutif a ébranlé l'appareil sécuritaire national, empêchant la législature et d'autres organismes gouvernementaux d'exercer un contrôle plus efficace sur le secteur de la sécurité. C'est ainsi que les vieilles habitudes de favoritisme perdurent au sein de lélite politique, entravant la bonne gouvernance et perpétuant la corruption. Les carences internes rendent la sécurité inopérante, exposant les citoyens à la petite corruption et aux abus de pouvoirs. Même si au niveau stratégique la politique s'est ouverte à la diversité, la société civile n'est toujours pas prise en compte et les corps législatif et judiciaire demeurent assujettis à 
l'exécutif. A moins d'une révision radicale de la constitution, le pouvoir exécutif continuera d'avoir plus d'influence sur le secteur de la sécurité que les deux autres branches du gouvernement, et la démocratie en souffrira.

Cependant, il faut mettre en perspective cette longue liste de tâches en attente, et l'apprécier par rapport aux progrès accomplis dans le secteur de la sécurité et à ceux qu'il reste à faire. Les progrès réalisés en matière de RSS doivent être évalués en comparaison de l'ampleur de la tâche tout entière. Une décennie est un laps de temps trop court pour modifier radicalement la dynamique de la gouvernance du secteur de la sécurité, et pourtant de nombreux résultats ont déjà été obtenus: par exemple, la loi de 2008 sur la défense nationale qui, avec la loi RSNR de 2011, représente une révision ambitieuse des principes juridiques régissant la prestation de services de sécurité, leur gestion et leur contrôle au Libéria.

En ce qui concerne le potentiel de transformation d'une RSS guidée par la gouvernance, l'aspect le plus significatif est sans doute le processus qui a conduit à ces changements. Outre la robustesse du nouveau cadre législatif, ce qui est remarquable est le fait qu'il a été établi sur la base de débats ouverts à tous et abordant tous les sujets, phénomène inconnu au Libéria auparavant. Il est d'ailleurs tout aussi exceptionnel que ces idées se soient concrétisées en un ensemble de lois élaborées par des législateurs nouvellement élus qui ont réussi à mener leur tâche à bien malgré les limitations existantes. Enfin, il faut noter que les progrès ont été réalisés dans un contexte pas vraiment propice à l'appropriation locale et qui ne faisait pas grand cas de la gouvernance démocratique au sein de programmes de RSS dirigés par des intervenants extérieurs. Ainsi, lévolution du Libéria symbolise le potentiel réalisable lorsqu'un soutien ciblé est apporté aux processus législatifs, tel que le renforcement des capacités parlementaires parmi les législateurs du secteur de la sécurité, lesquels ont pu ainsi améliorer la gouvernance. Cela montre aussi l'utilité de rendre la prise des décisions politiques plus transparente en y invitant la société civile et en encourageant les débats publics. On constate donc qu'un processus plus ouvert induit des propositions de réforme plus réactives, stimulant l'intérêt de tous pour la politique et favorisant la conciliation à propos des questions épineuses. La confiance de la population dans les nouveaux processus législatifs et dans la nouvelle organisation de l'ordre public s'en trouve accrue.

Il est impossible de prédire l'avenir, mais l'on a toutes les raisons dêtre optimiste et de penser que le Libéria soriente vers un secteur de la sécurité dans lequel le contrôle législatif et le contrôle civil sont en train de devenir réalité. Si la tendance se poursuit, le pays prendra un nouveau départ laissant derrière lui un long passé marqué par la suprématie présidentielle sur la sphère sécuritaire. Tout ce que le Libéria a vécu jusqu'ici corrobore ce nouveau sentiment de confiance en soi parmi les législateurs, qui devrait être encouragé, préservé, et stimulé par des débats publics de plus en plus nombreux et une pratique politique plus inclusive. 


\section{Notes}

${ }^{1}$ Pour les causes historiques de la crise de la gouvernance démocratique au Libéria, voir Jaye 2009.

${ }^{2}$ Selon William Barnes (2013), les trois règles de Tubman s'appliquaient aux fonctionnaires.

${ }^{3}$ La discussion suivante est inspirée du Rapport succinct sur les débats nationaux au sujet de la réforme du secteur de la sécurité au Libéria (Centre pour le contrôle démocratique des forces armées - Genève).

${ }^{4}$ La loi modifiant et abrogeant les paragraphes 1, 2, ainsi que le paragraphe 6(IV) de la loi de 2011 sur la sécurité nationale et les services de renseignement a été adoptée le 23 mai 2013 par l'honorable Chambre des représentants de la République du Libéria (Libéria 2013).

${ }^{5}$ Sur le rôle de la CRG, voir Jaye 2009.

${ }^{6}$ Entretien privé avec l'auteur, Monrovia, 2014. 